\title{
Ectopic expression of the beta-cell specific transcription factor Pdx1 inhibits glucagon gene transcription
}

\author{
B. Ritz-Laser ${ }^{1}$, B. R. Gauthier ${ }^{1}$, A. Estreicher ${ }^{1}$, A. Mamin'1, T. Brun 1 , F. Ris ${ }^{2}$, P. Salmon ${ }^{3}$, P. A. Halban ${ }^{2}$, \\ D. Trono ${ }^{3}$, J. Philippe ${ }^{1}$ \\ ${ }^{1}$ Diabetes Unit, University Hospital Geneva, Geneva, Switzerland \\ 2 Jeantet Research Laboratories, Geneva, Switzerland \\ ${ }^{3}$ Department of Microbiology, Centre Médical Universitaire, Geneva, Switzerland
}

\section{Abstract}

Aims/hypothesis. The transcription factor $\mathrm{Pdx} 1$ is required for the development and differentiation of all pancreatic cells. Beta-cell specific inactivation of $P d x l$ in developing or adult mice leads to an increase in glucagon-expressing cells, suggesting that absence of Pdx1could favour glucagon gene expression by a default mechanism.

Method. We investigated the inhibitory role of $\mathrm{Pdx} 1$ on glucagon gene expression in vitro. The glucagonoma cell line InR1G9 was transduced with a $P d x I$ encoding lentiviral vector and insulin and glucagon mRNA levels were analysed by northern blot and realtime PCR. To understand the mechanism by which Pdx1 inhibits glucagon gene expression, we studied its effect on glucagon promoter activity in non-islet cells using transient transfections and gel-shift analysis.

Results. In glucagonoma cells transduced with a $P d x I-$ encoding lentiviral vector, insulin gene expression was induced while glucagon mRNA levels were reduced by
50 to $60 \%$. In the heterologous cell line BHK-21, Pdx 1 inhibited by 60 to $80 \%$ the activation of the $\alpha$-cell specific element G1 conferred by Pax-6 and/or Cdx-2/3. Although Pdx 1 could bind three AT-rich motifs within G1, two of which are binding sites for Pax-6 and Cdx-2/3, the affinity of Pdx1 for G1 was much lower as compared to Pax-6. In addition, Pdx1 inhibited Pax6 mediated activation through G3, to which Pdx1 was unable to bind. Moreover, a mutation impairing DNA binding of Pdx1 had no effect on its inhibition on $\mathrm{Cdx}-2 / 3$. Since Pdx1 interacts directly with Pax-6 and Cdx-2/3 forming heterodimers, we suggest that Pdx 1 inhibits glucagon gene transcription through protein to protein interactions with Pax-6 and Cdx-2/3.

Conclusion/interpretation. Cell-specific expression of the glucagon gene can only occur when $P d x l$ expression extinguishes from the early $\alpha$ cell precursor. [Diabetologia (2003) 46:810-821]

Keywords Endocrine pancreas, $\mathrm{Pdx} 1, \mathrm{Pax}-6, \mathrm{Cdx}-2 / 3$, glucagon, transcriptional regulation, $\alpha$-cell.
The mammalian pancreas arises as two evaginations, the dorsal and ventral pancreatic anlage, from the embryonic endoderm in the region of the future duode-

Received: 21 January 2003 / Revised: 14 March 2003

Published online: 3 June 2003

(C) Springer-Verlag 2003

Corresponding author: B. Ritz-Laser $\mathrm{PhD}$, Diabetes Unit, University Hospital Geneva, 24, rue Micheli-du-Crest, 1211 Geneva 14, Switzerland

E-mail: Beate.Laser@medecine.unige.ch

Abbreviations: E, embryonic day; EMSA, electrophoretic mobility shift assay; GST, glutathione S-transferase; PD, paired domain; HD, homeodomain; PDHD, paired-linker-homeodomain. num. Determination of the pancreatic fate of the dorsal anlage requires signals from the notochord repressing endodermal Sonic hedgehog expression, whereas the ventral anlage forms independently from the notochord $[1,2]$. In the mouse, the first two transcription factors expressed in the prospective pancreatic endoderm are $H B 9$ and $P d x l$, which are detected at the 8 to 10 somite stage, respectively (embryonic day (E) 8-8.5) $[3,4,5]$; both factors are transiently expressed in the entire pancreatic anlage and are crucial for its development. Mice deficient in $H B 9$ selectively lack the dorsal pancreas due to a defect in specification of the pancreatic epithelium, whereas the ventral pancreas develops and exhibits more subtle defects in betacell differentiation and islet organization $[3,4]$. Inacti- 
vation of $P d x l$ results in pancreatic agenesis through a failure in proliferation of the pancreatic epithelium as well as in the branching and differentiation of the pancreatic buds $[5,6]$. The widespread expression of $H B 9$ and $P d x l$ in the embryonic pancreas declines at E9.5-10.5 (HB9) and E10.5 $(P d x 1)$ and they are later restricted to beta cells $(H B 9)$ or beta and $\delta$ cells $(P d x l)$. Similarly, the homeobox gene Isl-1 is transiently expressed in the entire dorsal bud and later restricted to pancreatic endocrine cells; mice deficient in Isl-1 lack the dorsal exocrine pancreas and have no differentiated islet cells $[7,8,9]$. Another transcription factor expressed very early (E9) during development in discrete cells of the pancreatic epithelium is $\mathrm{Pax}-6$, a member of the paired-homeobox family. Pax- 6 is crucial for the differentiation of glucagon-producing $\alpha$ cells inasmuch as Pax- 6 homozygous mutant mice have no or few $\alpha$ cells $[10,11]$. In contrast, inactivation of Pax-4, another paired homeobox gene, results in the selective lack of beta and $\delta$ cells; mice lacking both Pax-6 and Pax-4 have no endocrine cells [12].

Several lines of evidence suggest that $P d x l$ plays a key role not only in islet cell differentiation but also in maintaining the differentiated state and control of islet-hormone gene expression. Pdx1 is a major transactivator of the insulin and somatostatin genes through its synergistic interaction with E47/Beta2 and Pax6 or Pbx/Prep1, respectively $[13,14,15,16,17,18]$. Betacell specific inactivation of $P d x I$ in developing mice resulted in a decrease in beta-cells with a concomitant 2.5-fold increase in Glu+ cells and to coexpression of insulin and glucagon in $22 \%$ of cells suggesting that $\alpha$-cell differentiation and glucagon gene expression are favored by the absence of $P d x l$ in vivo [19]. Furthermore, transient inhibition of $P d x l$ in pancreatic beta cells of adult mice by antisense RNA expression using a Tet-On system led to reduction of $P d x l$ dependent beta-cell specific gene expression and to a striking increase in the number of glucagon gene expressing cells that were homogeneously distributed within the islet [20]. Similarly, we could recently show in a Tet-On system in insulinoma cells that functional inactivation of $P d x l$ resulted in differentiation of insulin-producing beta cells into glucagon-producing $\alpha$-cells [21].

In this work, we aimed to complement the studies of $P d x l$ function in beta cells by analysing the effect of ectopic $P d x l$ expression in $\alpha$ cells. Since $\alpha$-cell specificity is mainly characterized by glucagon gene expression, we studied $P d x l$ effects on glucagon gene transcription in glucagonoma (InR1G9) cells by Lentiviral transduction and on glucagon promoter activity in non-islet cells. We show a new potential function of $P d x l$ as mediator of cell-specific expression of the glucagon gene through inhibition of transcription.

\section{Materials and methods}

Cell culture and DNA transfection. The glucagon-producing hamster InRIG9 [22] and mouse $\alpha \mathrm{TC} 1$ [23], the insulin-producing hamster HIT-T15 [24], the non-islet Syrian baby hamster kidney (BHK-21) and human embryonic kidney (HEK) cell lines were grown in RPMI 1640 (Seromed; Basel, Switzerland) supplemented with $5 \%$ heat-inactivated fetal calf serum and 5\% heat-inactivated newborn calf serum, $2 \mathrm{mmol} / \mathrm{l}$ glutamine, $100 \mathrm{units} / \mathrm{ml}$ of penicillin, and $100 \mu \mathrm{g} / \mathrm{ml}$ of streptomycin. BHK-21 and HEK cells were transfected by the calcium phosphate precipitation technique [25] using 10-15 $\mu$ g of total plasmid DNA per $10-\mathrm{cm}$ petri dish. One $\mu \mathrm{g}$ of pSV2A PAP, a plasmid containing the human placental alkaline phosphatase gene, driven by the simian virus 40 (SV40) early promoter was added to monitor transfection efficiency [26]. Transfection of InR1G9 cells was done using the DEAE-dextran method as described previously [27]. cDNAs for the hamster $C d x-2 / 3$ and $P d x 1$ (M.S. German, University of California, San Francisco, Calif., USA), rat Isl-1 (D. Drucker, University of Toronto, Toronto, Canada), mouse Meis2 (N. Copeland, National Cancer Institute, Fredrick, USA), and quail Pax-6 (S. Saule, Institut Curie, Orsay, France), were cloned in the expression vector pSG5 (Stratagene). In experiments using variable quantities of expression vectors coding for $P d x 1, P a x-6$, or $C d x-2 / 3$, total amount of DNA was kept constant by adding appropriate amounts of the empty vector pSG5. Reporter plasmids consisted of the CAT reporter gene driven by different fragments of the rat glucagon gene promoter (-292GluCAT, -138GluCAT, G3-138GluCAT, G3-31GluCAT [28] or the rat insulin I gene promoter (-410InsCAT, comprising $410 \mathrm{bp}$ of the 5'flanking sequence and 49 bp of exon 1 and intron 1 of the rat insulin I gene) [29]. Data are presented as fold stimulation of the CAT activity obtained with the reporter plasmid alone and are the means +/- SEM of at least three experiments.

Transduction of InRIG9 cells with a lentiviral vector encoding $h P D X 1$. A Human $P D X 1$ cDNA $(h P D X 1)$, kindly provided by V. Schwitzgebel (University Hospital Geneva, Geneva, Switzerland), was subcloned into pBluescript (Stratagene) and then inserted into an optimized HIV-based vector (pWPT, kindly provided by M. Wiznerowicz, Faculty of Medecine, Geneva, Switzerland), generating the pWPT-PDX transfer plasmid. Map and sequence are available at http://www.medecine.unige.ch/ salmon/pWPT-PDX.html. Stocks of pWPT-PDX lentiviral vectors were produced using transient cotransfection of 293 T cells with pWPT-PDX, R8.91 (second generation HIVpackaging plasmid [30], and pMDG (VSV envelope-expression plasmid) as described previously [31]. Vectors were concentrated by ultracentrifugation and resuspended in serum-free medium (CellGro SCGM, CellGenix, Freiburg, Germany) as described previously [32]. Vector stocks were stored at $-70^{\circ} \mathrm{C}$ and titres were determined by enzymatic assay of HIV reverse transcriptase. For hPDX1 transduction, $3 \times 10^{4}$ INR1G9 cells were transduced in $50 \mu \mathrm{l}$ of RPMI $164010 \%$ FCS with $2.5 \times 10^{4}$ pPWT-PDX transducing units (as determined by RT assay). After $24 \mathrm{~h}$, cells were washed twice with RPMI $10 \%$ FCS and cultured for further $72 \mathrm{~h}$ before testing for $P d x 1$ expression by immunocytochemistry using anti-Pdx 1 antibodies kindly provided by C. V. E. Wright (Vanderbilt Medical Center, Nashville, Tenn., USA). RNA was isolated for northern blotting using Trizol (Invitrogen) and whole-cell protein extracts were prepared for EMSA. Northern blots were sequentially hybridized with random labelled complementary DNA (cDNA) probes for rat glucagon and proinsulin I and an $18 \mathrm{~S}$ ribosomal (rRNA) oligonucleotide probe (5'-GCCGTCCCTCTTAATCATGGCCTCAGTTCC). Hybridization signals of the 
pWPT-PDX transduced InR1G9 cells were quantified using a PhosphoImager (Molecular Dynamics) and expressed as glucagon mRNA/18S rRNA ratio relative to the control cells.

Quantitative RT-PCR. Reverse transcription was done by using $2 \mu \mathrm{g}$ of total RNA isolated from HIT-T15 and from non-infected or lentivirus-infected InR1G9 cells, random hexanucleotide primers and Superscript II reverse transcriptase (Invitrogen). One tenth of the resulting cDNA was used for real-time PCR in the LightCycler instrument (Roche) using the QuantiTect SYBR Green PCR kit (Qiagen) as recommended by the provider. The following primers were used: insulin sense $5^{\prime}$ tcttctacacaccaagacc, antisense $5^{\prime}$-gttccacaatgccacgcttc; glucagon sense $5^{\prime}$-gatcattcccagcttcccag, antisense $5^{\prime}$-ctggtaaaggtcccttcagc; 28S rRNA sense 5'-tagccaaatgcctcgtcatc, antisense 5'acctctcatgtctcttcacc.

Chloramphenicol aetyltransferase (CAT) and protein assays. Cell extracts were prepared $48 \mathrm{~h}$ after transfection and analysed for CAT and alkaline phosphatase activities as described previously [28]. Quantification of acetylated and non-acetylated forms was done with a PhosphorImager (Molecular Dynamics). A minimum of three independent transfections was done; each of them carried out in duplicate.

Electrophoretic Mobility Shift Assays (EMSAs). Nuclear extracts from InR1G9, HIT-T15 or BHK-21 cells transfected with $P a x-6$ or $P d x l$ expression plasmids or the empty vector pSG5 were prepared according to [33]. EMSAs were carried out as described previously [34] adding first 8 to $28 \mu \mathrm{g}$ ( $8 \mu \mathrm{g}$ in standard reactions) of nuclear extracts and subsequently labelled oligonucleotides containing the rat glucagon gene G1 (G1-56, G1-52, G1-54, G1-50) or G3 (5'-GCTGAAGTAGTTTTTCACGCCTGACTGAGATTGAAGGGTGTATTTC) sequence $[35,36]$, or the A3/A4 element of the rat insulin I gene (InsA3/A4, 5'-GATCTTGTTAATAATCTAATTACCCTAGAACAATTATTAGATTAATGG) [37]. In competition experiments, labelled and cold oligonucleotides were added simulaneously and when specific antibodies were used, they were added after half of the incubation time. Antisera were generously provided by $\mathrm{H}$. Edlund (anti-Pdx 1; University of Umea, Umea, Sweden), S. Saule (anti-Pax-6 homeodomain, serum no. 13; Institut Curie, Orsay, France), D. Melloul (anti full-length Pdx1; Hebrew University Hadassah Medical Center, Jerusalem, Israel), and M.S. German (anti-Cdx-2/3; University of California, San Francisco, Calif., USA).

Mutagenesis of Pdxl. In vitro mutagenesis was done by using the QuikChange Site-Directed Mutagenesis Kit (Stratagene, The Netherlands) according to the manufacturer's protocol using the following primers for the I192Q mutation of $P d x 1$ : forward $5^{\prime}$-gagacacatcaagcagtggttccaaaacc and reverse $5^{\prime}$-ggttttggaaccactgcttgatgtgtctc.

GST fusion proteins and GST-precipitation. Hamster Pax-6 GST fusion proteins have been described [36]. GST fusion proteins were expressed in E. coli and purified according to the manufacturer recommendations (Pharmacia). L-[ $\left.{ }^{35} \mathrm{~S}\right]$ methionine-labelled Pdx1 was generated in vitro using the TNT wheat germ extract system (Promega) and GST-precipitations were done as described previously [36].

Data analysis. Data are presented as means \pm SE and statistical significance was tested by analysis of variance and Student's $t$ test where applicable. The threshold for statistical significance was a $p$ value of less than $0.05(*$ and $* *$ indicate statistical significance with $p<0.05$ and $p<0.01$, respectively).

\section{Results}

Lentiviral transduction of $P d x 1$ in InRIG9 cells inhibits transcription of the endogenous glucagon gene. To assess the effect of ectopic $P d x l$ expression in glucagonproducing cells, we transduced the hamster glucagonoma cell line InR1G9 with a lentiviral vector coding for $h P d x l$. After 4 days, expression of Pdx 1 in two independent transductions of InR1G9 cells was confirmed by immunocytochemistry and EMSA in $90 \%$ of the cells (data not shown). Northern blot and real-time PCR analysis showed that $P d x l$ repressed endogenous glucagon mRNA levels by $50 \%$ and induced the endogenous insulin gene in the glucagonoma cell line (Fig. 1).

Pdxl inhibits transcriptional activation of the glucagon gene promoter by Pax-6 and $C d x-2 / 3$. To investigate the mechanisms by which $P d x l$ decreases glucagon mRNA levels, we analysed its effect on transactivation of glucagon gene reporter constructs in the non-islet cell line BHK-21. The paired-homeodomain protein Pax-6, which is essential for the development of $\alpha$ cells, acts as a major transactivator of the glucagon gene by its interaction with the $\alpha$-cell-specific promoter element $\mathrm{G} 1$ and the enhancer G3 [10, 11, 36]. In addition, the homeodomain protein $\mathrm{Cdx}-2 / 3$ transactivates the glucagon gene promoter independently through G1 and acts synergistically with Pax-6 $[36,38]$. Since Pdx1 has been reported to interact with both Pax-6 and Cdx-2/3 in vitro $[15,38]$, we investigated the effects of Pdx 1 on Pax-6 and $\mathrm{Cdx}-2 / 3$ induced activation of glucagon gene promoter constructs. Overexpression of $P d x l$ resulted in a dosedependent inhibition, up to $90 \%$, of Pax-6 mediated transcriptional stimulation whether G1 (-138GluCAT), G3 (G3-31GluCAT), or both G1 and G3 (G3-138GluCAT) were present in the reporter construct (Fig. 2). Pdx 1 is thus able to inhibit Pax-6 mediated activation of glucagon gene expression through both the G1 and G3 elements in BHK-21 cells. In addition to its inhibitory action on Pax-6, Pdx 1 reduced the $\mathrm{Cdx}-2 / 3$ and Pax6/Cdx-2/3-mediated activations of G1 (-138GluCAT) by $65 \%$ and $85 \%$, respectively (Fig. 2C). Control cotransfections with Isl-1 [39] and Meis2 [40] had no effect on Pax-6 or Cdx-2/3 induced transcriptional activation (Fig. 2C).

When $P d x l$ was transfected in BHK-21 cells in the absence of Pax- 6 and $C d x-2 / 3$, CAT activity driven by -138 Glu was stimulated (Fig. 2C, Fig. 3). This effect was dose-dependent and quantitatively similar to the activation observed with the rat insulin I promoter construct -410InsCAT (Fig. 3). However, the maximal 4.6-fold stimulation of the glucagon gene promoter by $P d x l$ was weak when compared to that obtained with Pax-6 or $P a x 6 / C d x-2 / 3$.

Pdxl binds to the G1 element. The G1 element of the glucagon gene promoter contains three AT-rich se- 
A

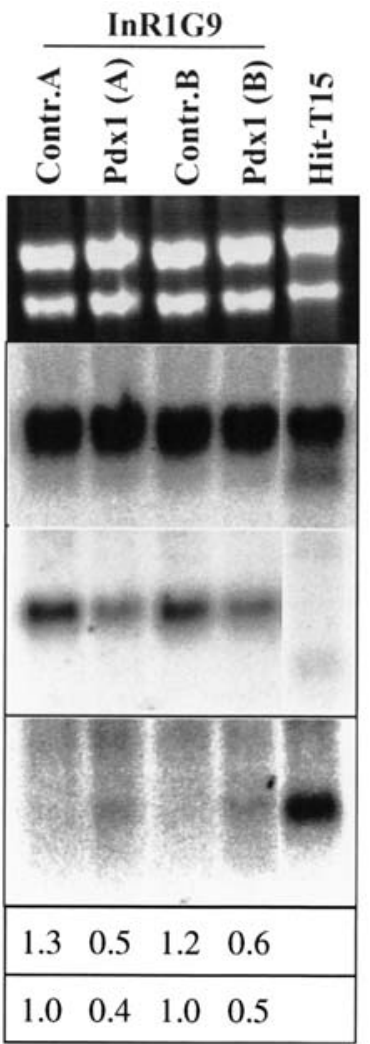

28S

$18 \mathrm{~S}$

\section{S rRNA}

\section{Glucagon}

\section{Insulin}

Ratio glucagon/18S rRNA

Relative glucagon mRNA level

B 120

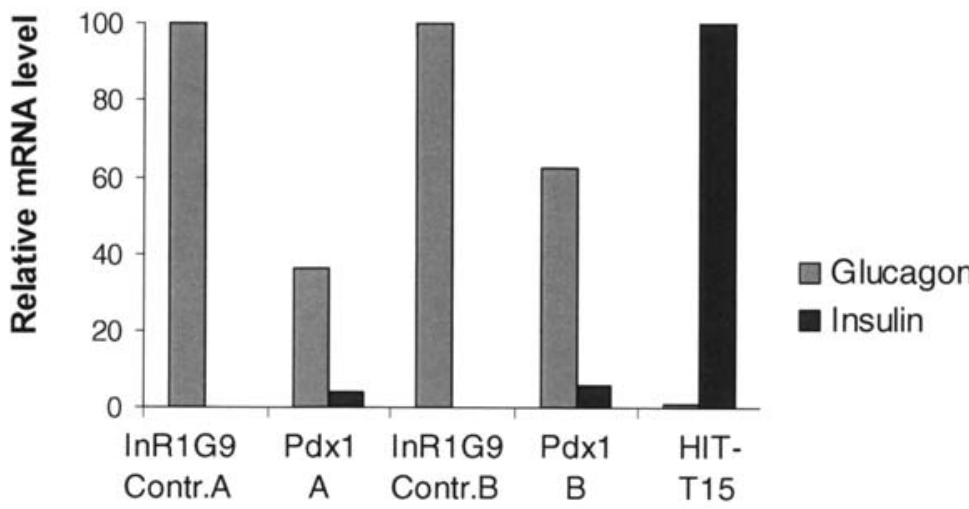

Fig. 1A, B. Pdx1 induces insulin mRNA and reduces endogenous glucagon mRNA levels in glucagonoma cells. Northern blot (A) and Real-time RT-PCR (B) analysis of glucagon-producing InR1G9 cells (contr. A, contr. B), from two experiments of unselected InR1G9 cellpools transduced with a lentiviral vector coding for $h P d x l(\mathrm{Pdx} 1 \mathrm{~A}, \mathrm{Pdx} 1 \mathrm{~B}$,), and Hit-T15 cells. The proportion of infected cells was roughly $90 \%$ (immunocytochemistry), but Pdx1 infected cellpool A showed a slightly stronger Pdx1 protein complex in EMSA that cellpool B. (A) Northern blot sequentially hybridized with probes for insulin, glucagon and 18S rRNA. (B) Quantitative RT-PCR showing that $P d x l$ reduces glucagon mRNA levels to roughly $36 \%$ and $62 \%$ of wild-type levels (cellpool A and B, respectively). Whereas insulin mRNA was undetectable in noninfected InR1G9 cells by Northern blot (A), quantitative RT-PCR provided evidence of as little as $0.2 \%$ of insulin mRNA levels found in HIT-T15 cells. Pdx1 infection increased insulin mRNA levels by 21 and 27-fold reaching $4 \%$ and $6 \%$ (cellpool $\mathrm{A}$ and $\mathrm{B}$, respectively) of HIT-T15 insulin mRNA levels quences, two of which form a nearly perfect direct repeat with binding sites for the homeodomain containing proteins Pax-6 and Cdx-2/3 (Fig. 4A) [35, 36, 38, 41]. Since $P d x l$ is a member of the Antennapedia family of homeodomain proteins [42] and able to transactivate $-138 \mathrm{Glu}$ in the absence of Pax-6 and $C d x-2 / 3$, we tested whether Pdx 1 could bind to the glucagon gene promoter. With glucagon-producing InR1G9 cell extracts, at least three protein complexes are formed on G1-56 comprising the direct repeat element within G1; Pax-6 as a monomer, Pax-6/Cdx-2/3 as a heterodimer, and HNF3 $\beta$ [36, 43] (Fig. 4A,B, G1-56). Using nuclear extracts from the insulin-producing cell line HIT-T15, which contains Pdx1, an additional complex was detected. This band co-migrated with a complex formed in BHK-21 cells overexpressing $P d x I$ and was recognized by anti-Pdx1 antibodies. 
$\mathbf{A}$

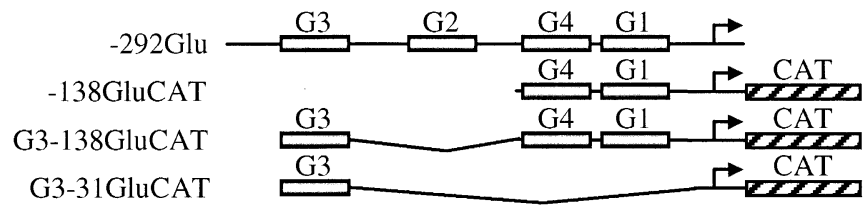

B

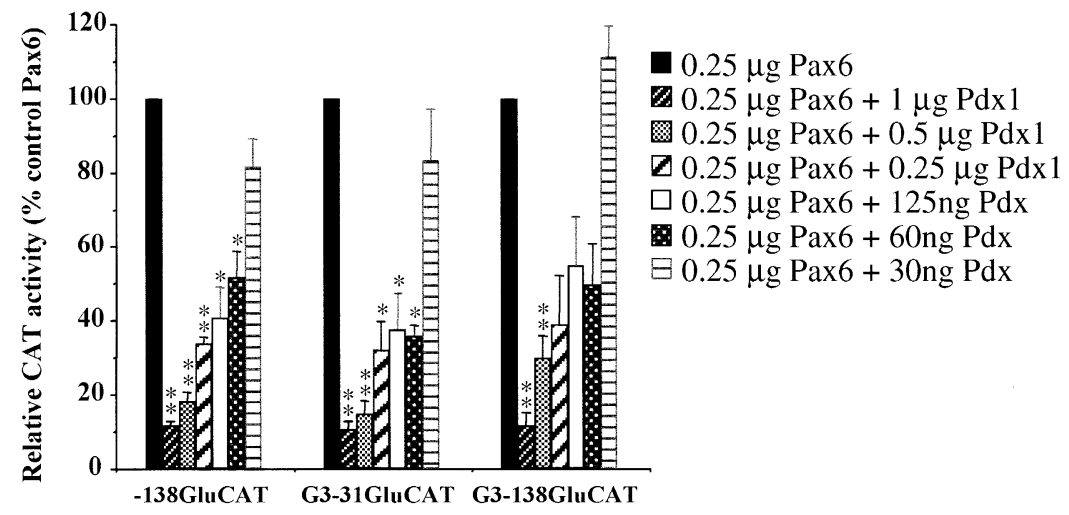

C

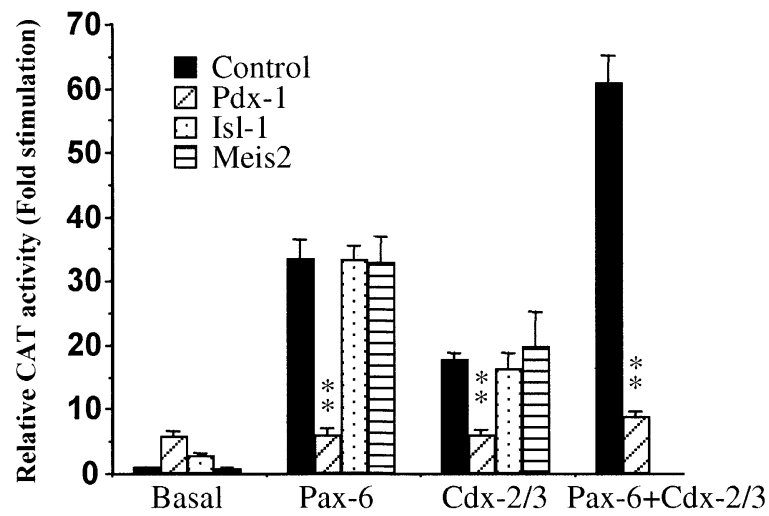

-138 Glu CAT

Fig. 2A-C. $P d x l$ interferes with transcriptional activation of the glucagon gene promoter by $P a x-6$ and $C d x-2 / 3$. (A) Schematic representation of glucagon reporter gene constructs used in this study. Characterized cis-acting control elements are designated as G1 to G4. (B) Dose-dependent inhibition of the Pax-6-mediated activation of the glucagon gene elements G1 (-138GluCAT) and/or G3 by $P d x 1$ in BHK-21 cells. (C) To assess their effect on $P a x-6$ and/or $C d x-2 / 3$ mediated activation of the glucagon gene promoter, BHK-21 cells were cotransfected with $1 \mu \mathrm{g}$ of $P d x I, P a x-4$, Meis2, and/or Isl-1 expression vectors, $250 \mathrm{ng}$ of expression vectors containing the Pax-6 and/or $C d x-2 / 3$ cDNAs and $10 \mu \mathrm{g}$ of -138 GluCAT

Pdx1 is thus able to interact with the G1 element. To localize the binding site of $\mathrm{Pdx} 1$ within G1, we used oligonucleotides G1-54, G1-52, and G1-50 representing the three AT-rich elements. Pdx1 bound to all these sites (Fig. 4B) and is thus capable of interacting with three AT-rich motifs within the first $100 \mathrm{bp}$ of the glucagon gene promoter.

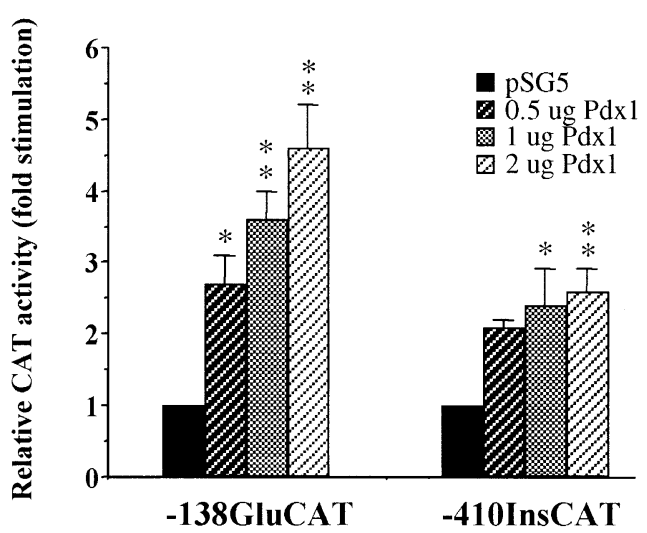

Fig. 3. Dose-dependent activation of the glucagon gene element G1 by $P d x 1$. BHK-21 cells were cotransfected with $10 \mu \mathrm{g}$ of the indicated reporter plasmids and increasing amounts of $P d x l$ expression vectors 


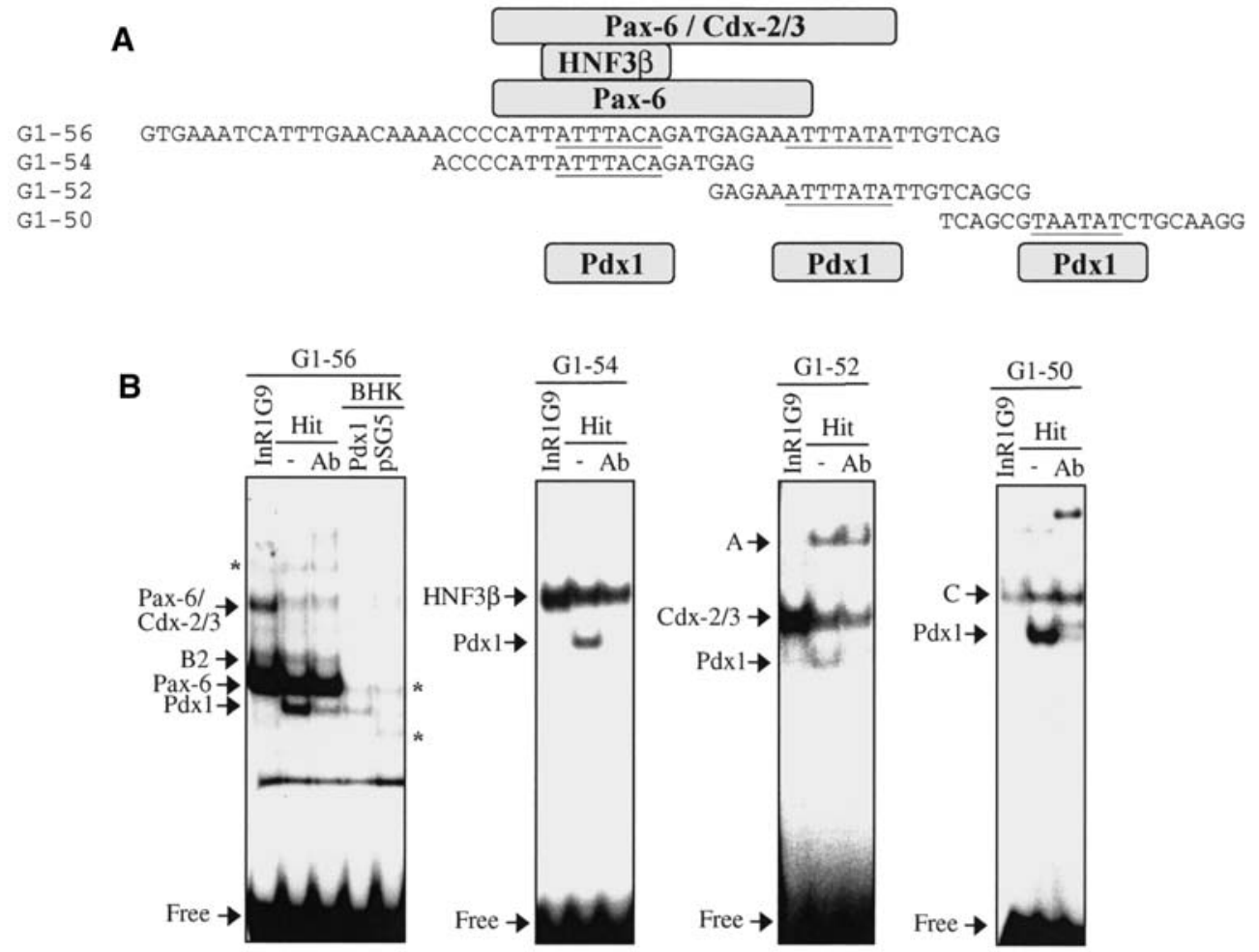

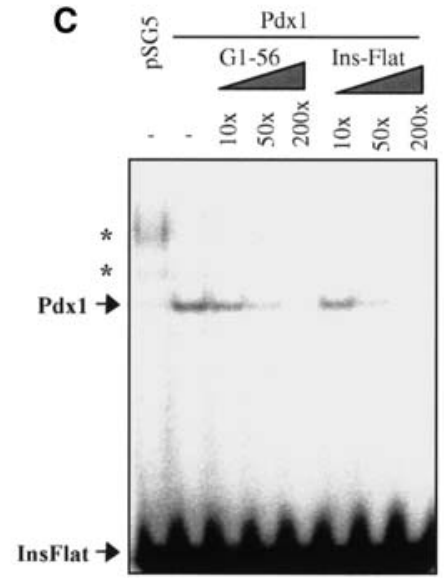

Fig. 4A-D. Pdx 1 binds to three sites within the glucagon gene G1 element. (A) Schematic diagram illustrating oligonucleotides for the glucagon gene G1 element and protein complexes formed on G1. The AT-rich elements are underlined. (B) EMSA using nuclear extracts from InR1G9, Hit-T15, or BHK-21 cells overexpressing $P d x 1$. Pdx 1 was able to bind the three AT-rich elements contained within G1. $A b$ indicates the addition of antiPdx1 antibodies. A and $\mathrm{C}$ represent yet unidentified proteinDNA complexes. $(\mathbf{C}, \mathbf{D})$ Nuclear extracts of BHK-21 cells overexpressing $P d x 1$ were incubated with the InsA3/A4 or G1-50 oligonucleotide and the indicated excess of cold competitor oligonucleotides. Bold arrowheads and asterisks indicate specific and non-specific complexes, respectively

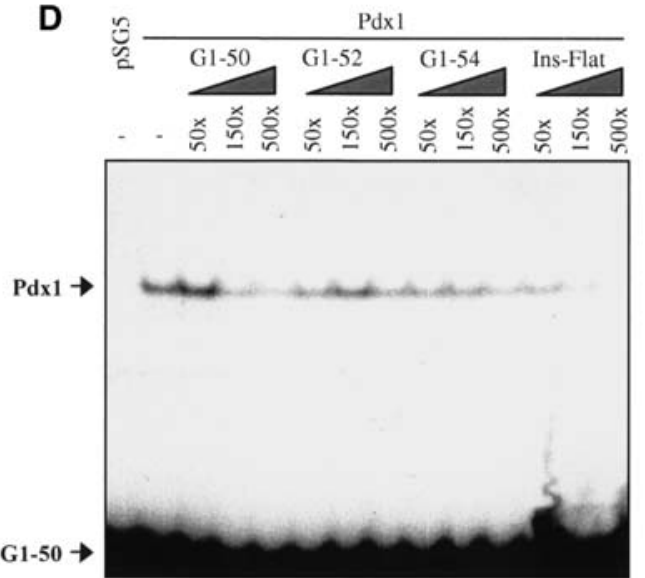

To assess the relative affinity of Pdx 1 for G1 compared to the A3/A4 of the rat insulin I gene, a known Pdx1 binding site, we carried out EMSA competition experiments. A similar molar excess of cold G1-56 and InsA3/A4 oligonucleotides competed for the Pdx1 complex formed with InsA3/A4 indicating that Pdx1 binds to both elements with roughly the same affinity (Fig. 4C). These data are in agreement with the equivalent transactivation of the glucagon and insulin gene promoters by $P d x l$. When the individual glucagon gene binding sites were used for EMSA, the most proximal motif G1-50 displayed a slightly higher affinity for Pdx 1 compared to G1-52 and G1-54 located within G1-56 but lower compared to InsA3/A4 (Fig. 4D). 
A

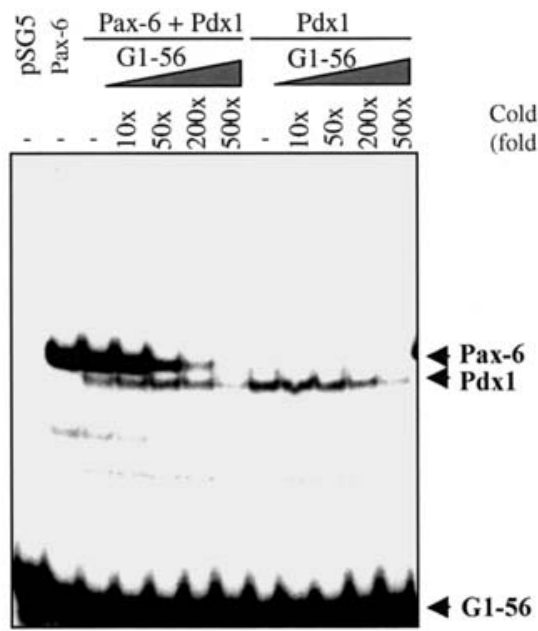

B

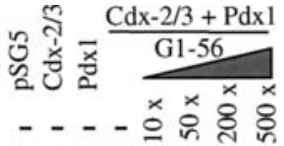

Cold Competitor fold excess)

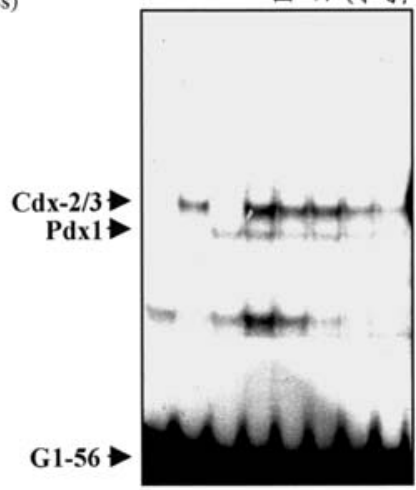

\section{C}

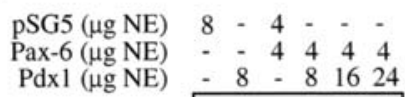

$\mathrm{Pdxl}(\mu \mathrm{g} \mathrm{NE})-8-81624$

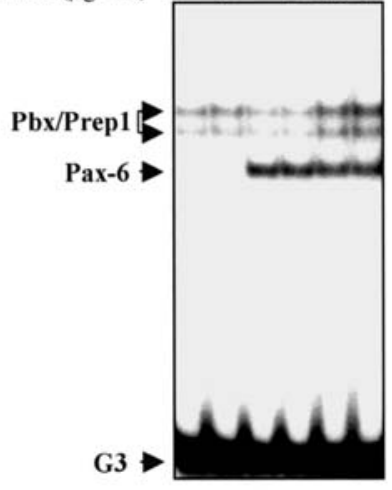

E

pSG5 ( $\mu \mathrm{g} \mathrm{NE}) \quad-\quad-\quad-4$ $\mathrm{Cdx}-2 / 3(\mu \mathrm{g}$ NE) $4-4 \quad 4 \quad 4$ Pdxl ( $\mu \mathrm{g} \mathrm{NE})$ - 44444 -

Antibody

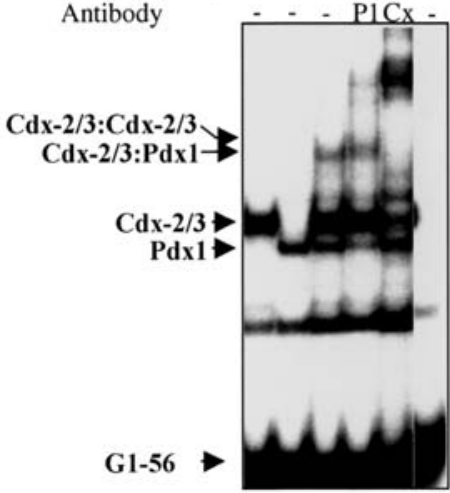

D

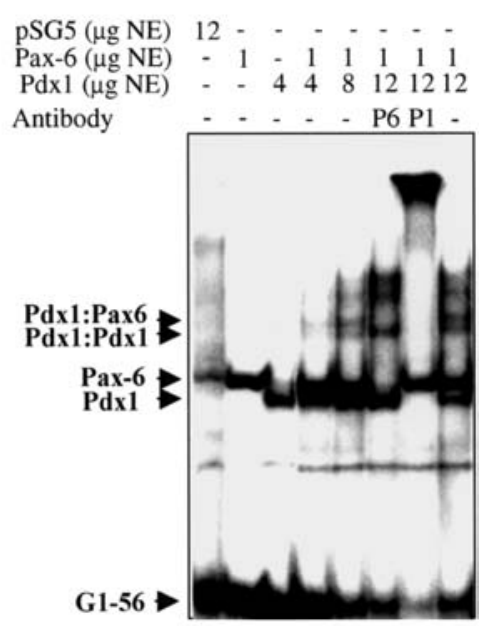

Fig. 5A-E. Pdx 1 does not impair Pax-6 binding to the glucagon gene promoter. (A, B) Competition experiments analysing the relative affinity of Pdx 1 and Pax- 6 for the G1 element. Protein-DNA complexes formed with nuclear extracts from BHK21 cells overexpressing $P d x 1, P a x-6$ or $C d x-2 / 3$ and G1-56 were competed for by the indicated molar excess of cold oligonucleotides. (C) Pax-6 containing extracts from BHK-21 cells were mixed with increasing amounts of Pdx1 containing extracts and incubated with the G3 oligonucleotide. No impairment of Pax- 6 binding by Pdx 1 was observed. Heterodimers of the widely expressed Prep1/Pbx proteins formed on G3 [44] are observed in all BHK-21 extracts. (D) Pdx1 formed a heterodimer with Pax-6 on G1-56; this complex was displaced by adding anti-Pax6 (P6) or anti-Pdx1 (P1) antibodies. The identity of two further slower migrating complexes containing Pdx 1 has yet to be determined. (E) When Pdx1 containing extracts were mixed with $\mathrm{Cdx}-2 / 3$ containing extracts, $\mathrm{Cdx}-2 / 3$ formed a heterodimer with $\mathrm{Pdx} 1$ on G1-56; this complex was displaced by adding anti-Cdx-2/3 antibodies. Note that when anti$\mathrm{Pdx} 1$ antibodies were used, the $\mathrm{Cdx}-2 / 3-\mathrm{Pdx} 1$ heterodimer was displaced and a $C d x-2 / 3$ homodimer with only slightly slower migration than $\mathrm{Cdx}-2 / 3-\mathrm{Pdx} 1$ was formed 
$P d x 1$ forms heterodimers with Pax-6 and $C d x-2 / 3$ on G1. To investigate the mechanism by which $\mathrm{Pdx} 1$ affects the Pax- 6 and Cdx-2/3 mediated activation of the glucagon gene, we assessed whether Pdx 1 could affect Pax-6 and Cdx-2/3 binding to DNA in vitro. When BHK-21 nuclear extracts containing Pax- 6 were mixed with Pdx-containing extracts, Pdx1 formed a weaker complex on G1-56 as compared with the binding reaction without Pax-6 (Fig. 5A). Furthermore, competition with cold G1-56 showed a better affinity of Pax-6 compared with Pdx1 for G1. By contrast with Pax-6, Cdx-2/3 and Pdx1 displayed similar affinity for G1-56 (Fig. 5B).

Alternatively to a mechanism of direct competition for the binding site, Pdx 1 could interact with Pax-6 and/or Cdx-2/3 and either form a heterodimer or impair DNA binding by protein to protein interaction. We therefore analysed the effect of adding various amounts of Pdx1 on Pax-6 binding to G3 and G1. Pdx1 overexpressed in BHK-21 cells did not interact with G3, only heterodimers of the ubiquitous proteins $\mathrm{Pbx}$ and Prep1 [44] were observed (Fig. 5C). Pax-6 formed a complex with G3 that was not affected by the addition of increasing amounts of $\mathrm{Pdx} 1$-containing extracts (Fig. 5C). In contrast to G3, Pdx1 bound as a monomer and a homodimer to G1 (Fig. 5D). In addition, a Pax6:Pdx1 heterodimer was formed that was recognized by both anti-Pdx 1 and anti-Pax- 6 antibodies, thus confirming the protein to protein interaction of both transcription factors (Fig. 5D). When Pdx1 and $\mathrm{Cdx}-2 / 3$ containing extracts were mixed, both proteins bound as monomers and $\mathrm{Pdx} 1$ did not impair $\mathrm{Cdx}-2 / 3$ binding. In addition, both proteins formed a heterodimer that was recognized by anti-Cdx-2/3 and anti-Pdx 1 antibodies (Fig. 5E). Note that when anti$\mathrm{Pdx} 1$ antibodies were used, the Cdx-2/3-Pdx1 heterodimer was displaced by a Cdx-2/3 homodimer with only slightly slower migration than $\mathrm{Cdx}-2 / 3-\mathrm{Pdx} 1$. We therefore conclude that $\mathrm{Pdx} 1$ could exert its transcriptional inhibition on the glucagon gene promoter through protein to protein interaction with Pax- 6 and $\mathrm{Cdx}-2 / 3$ that could change their conformation and interfere with their transcriptional properties. Furthermore, inhibition of Pax-6 mediated activation could also in part be mediated by competition of binding to the G1 but not the G3 element.

Pdxl interacts with the homeodomain of Pax-6. To test for direct physical interactions of Pdx1 and Pax-6, we did GST precipitation assays using GST fusion proteins containing either the Pax-6 paired domain (PD), homeodomain (HD) or paired-linker-homeodomain (PDHD) and ${ }^{35} \mathrm{~S}$-labelled Pdx1. Protein to protein interactions with Pdx1 were observed with fusion proteins comprising the Pax-6 HD (10\% of the input), whereas precipitation of Pdx 1 with the Pax-6 PD was weak. However, adding the PD and linker domain to the Pax-6 HD increased interaction to $17 \%$ (Fig. 6).
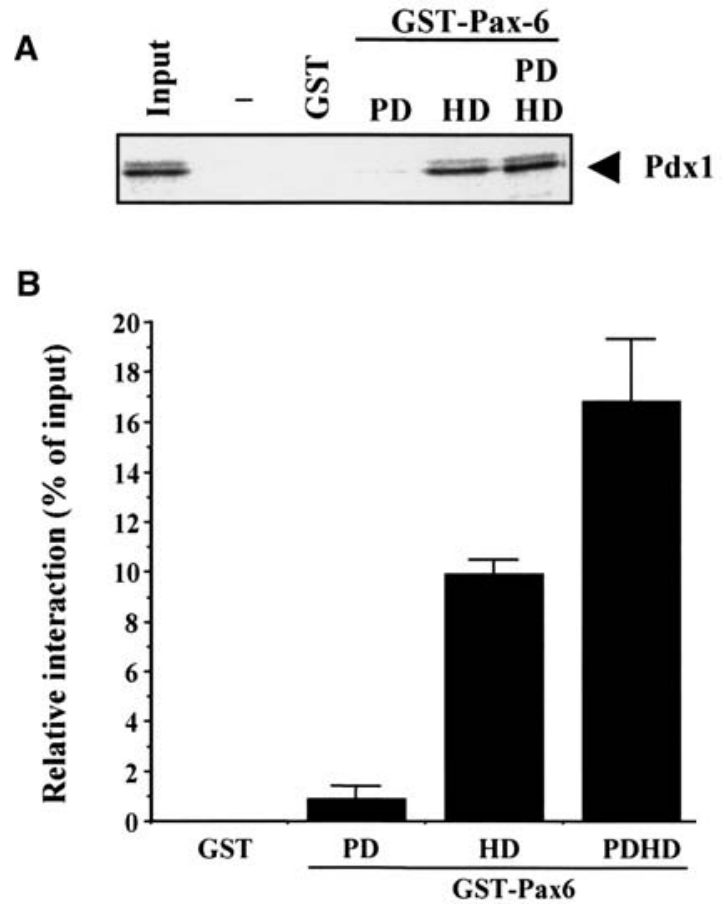

Fig. 6A, B. Pdx1 interacts with the HD of Pax-6. (A) GST-precipitation assay using $10 \mu \mathrm{g}$ of GST alone, GST-Pax-6 paired (PD), homeo (HD) or paired-linker-homeodomain (PDHD, fusion proteins immobilized on sepharose beads and in vitro synthesized, ${ }^{35} \mathrm{~S}$-labelled $\mathrm{Pdx} 1$. Lane input, $10 \%$ of the respective in vitro translation reaction used for protein to protein interaction. (B) Quantification of protein interactions shown in (A) as the precipitation rates relative to the respective input. Data are presented as the mean \pm SEM of at least three experiments

These data show that Pdx 1 interacts with Pax-6 in the absence of DNA and that major contacts are mediated by the Pax- 6 homeodomain although the linker domain and/or PD might be required for full interactions.

Inhibition of Pax-6 and Cdx-2/3 mediated activation is independent of PdxI DNA binding. To further test our hypothesis that Pdx 1 represses Pax- 6 and Cdx-2/3 mediated activation of the glucagon gene promoter through protein to protein interactions, we mutated $P d x l$ in an amino acid (I192Q) of the DNA recognition helix within the homeodomain that has been reported to abolish binding to the A3/A4 element of the rat insulin I gene promoter [45]. This mutant Pdx1 protein was produced at equivalent levels in fibroblast cells and in in vitro translation assays (Fig. 7A), and its binding activity to the G1 element was reduced by more than $90 \%$ (Fig. 7B).

In cotransfection assays, a quantitatively similar repression of $\mathrm{Cdx}-2 / 3$ was observed with the $\mathrm{Pdx} 1 \mathrm{wt}$ and mutant I192Q protein (Fig. 7C) confirming that the inhibition of $\mathrm{Cdx}-2 / 3$ mediated transcriptional activation is independent of Pdx1 DNA binding and rather occurs through protein to protein interactions. In contrast, repression of Pax-6 mediated activation was attenuated by the Pdx 1 mutation (78\% and 54\% 
A

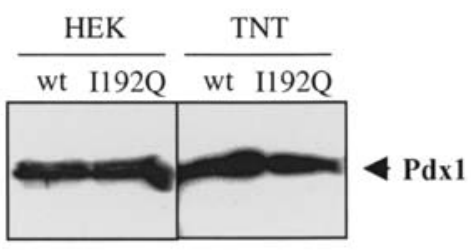

B

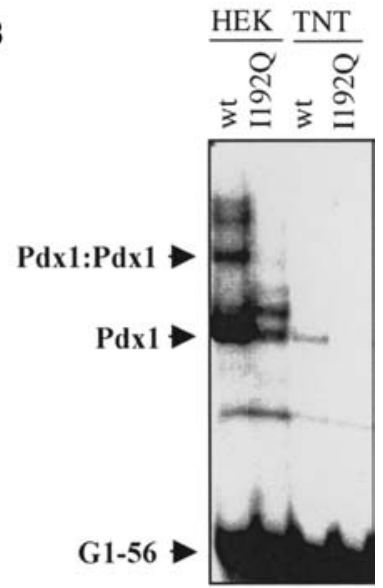

C

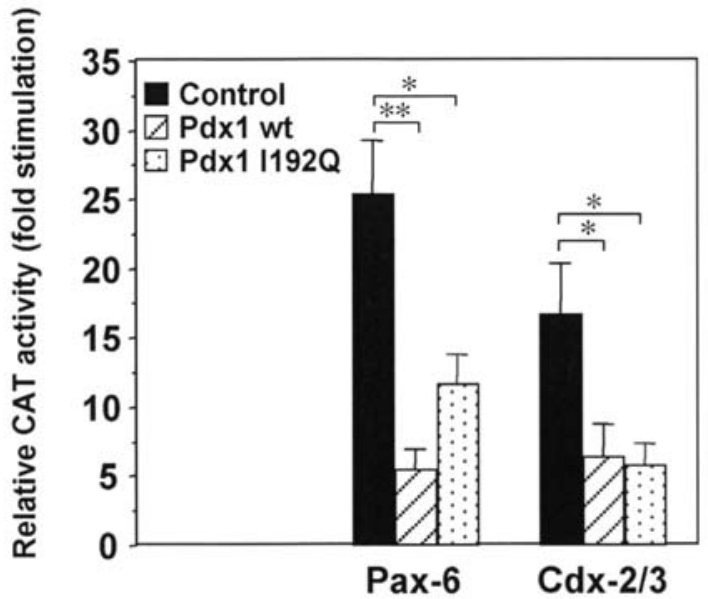

D

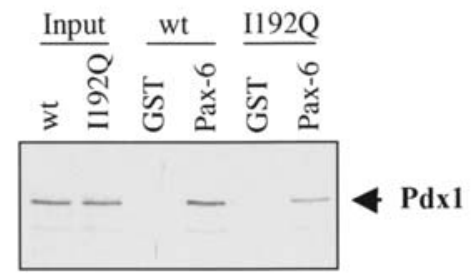

Fig. 7A-D. Mutant Pdx1 protein impaired in DNA binding still repressed transcriptional activation of the glucagon gene promoter by Pax6 and $\mathrm{Cdx}-2 / 3$. (A) Western blot analysis showing similar synthesis of wild-type (wt) and mutant (I192Q) Pdx1 in HEK cells and in in vitro transcription/translation (TNT). (B) EMSA analysis showing 90\% reduced binding activity of Pdx1 I192Q to G1 as compared to Pdx1 wt. (C) To assess the effect of mutant $P d x I$ on Pax- 6 and $C d x-2 / 3$ mediated activation of the glucagon gene promoter, BHK-21 cells were cotransfected with $1 \mu \mathrm{g}$ of wt $P d x$ or $P d x 1$ I192 $Q$ expression vectors and $250 \mathrm{ng}$ of expression vectors containing the Pax-6 or $C d x-2 / 3$ cDNAs as well as $10 \mu \mathrm{g}$ of -138 GluCAT. (D) GST-precipitation assay using $10 \mu \mathrm{g}$ of GST alone or GSTPax-6 paired-linker-homeodomain fusion protein immobilized on sepharose beads and in vitro synthesized, ${ }^{35} \mathrm{~S}$-labelled $\mathrm{Pdx} 1$ wt or I192Q. Lanes input, $10 \%$ of the respective in vitro translation reactions used for protein to protein interaction by wt and I192Q Pdx1, respectively). We therefore tested by GST pulldown whether the I192Q mutation affected interactions between Pdx 1 and Pax-6. Protein to protein interactions of both transcription factors were reduced by approximately $65 \%$ indicating that the Pdx 1 homeodomain is critical for protein interactions with Pax-6 (Fig. 7D). Since the reduced repressor activity of Pdx1 I192Q on Pax-6 corresponds quantitatively with the decreased protein to protein interaction of both factors, we suggest that Pdx 1 could exert its repressor activity primarily through protein to protein interaction. Our data cannot, however, rule out a contribution of DNA binding competition to its inhibitory effect on Pax-6.

\section{Discussion}

We show that $P d x l$ is able to act as a repressor of the endogenous glucagon gene in InR1G9 glucagonoma 
cells while simultaneously inducing insulin gene transcription. Similarly, $P d x l$-induction of insulin and islet-amyloid-polypeptide gene expression has been shown in stable clones of glucagon-producing AN697 and $\alpha \mathrm{TC} 1$ cells, but in these studies, no effect on glucagon gene expression has been observed [46, 47]. Although we have no clear explanation for this discrepancy, our recent results showing de-repression of the glucagon gene through overexpression of a dominantnegative Pdx1 protein in insulinoma cells [21] and in vivo data showing de-repression of glucagon gene expression by functional inactivation of $P d x l$ in beta cells of developing and adult mice $[19,20]$ support our findings. However, these studies do not discriminate between direct effects of $P d x l$ on glucagon gene expression and indirect effects mediated by the transcriptional network of the beta cell. Overexpressing $P d x 1$ in glucagonoma cells allowed us to assess the role of Pdx1 on the relative expression of the insulin and glucagon genes in the absence of other beta-cell specific factors and demonstrates the critical direct role of Pdx1 in the cell's ability to transcribe these genes.

The inhibitory effect of Pdx 1 on glucagon gene expression is mediated in vitro by impairing Pax-6 and $\mathrm{Cdx}-2 / 3$ driven activation of the $\mathrm{G} 3$ and $\mathrm{G} 1$ promoter elements. Pdx 1 did not interact directly with G3 and although it bound to three AT-rich elements within G1, two of which are binding sites for Pax-6 and $\mathrm{Cdx}-2 / 3$, its relative affinity was lower as compared to Pax-6. Pdx1 is subject to post-translational modifications such as phosphorylation altering its subcellular localization [48]. We cannot exclude that these modifications could enhance Pdx1 binding to the G1 element in vivo. However, the DNA binding deficient Pdx 1 mutant I92Q repressed Cdx-2/3 mediated activation quantitatively similarly to the wild-type protein and although Pax-6 driven activation was reduced with Pdx1 I192Q, protein-to-protein interactions with Pax-6 were equally affected. Moreover, since Pdx 1 repressed Pax- 6 mediated activation also through G3, a paired box binding site, we suggest that the major molecular mechanism of transcriptional inhibition by $\mathrm{Pdx} 1$ could be through protein to protein interaction although we cannot exclude a contribution of DNA binding competition to its inhibitory effect on Pax-6.

Two mechanisms might thus explain the repression of transcriptional activation by $\mathrm{Pdx} 1$; (i) through direct interactions with Pax-6 and Cdx-2/3, Pdx 1 could change their conformations thereby impairing contacts to coactivator proteins or favour contacts with corepressors. We show by GST precipitation assays protein to protein interactions between $\mathrm{Pdx} 1$ and Pax-6 that are mediated in part by the Pax- 6 homeodomain and the capacity of Pdx 1 to form a heterodimer with both Pax-6 and Cdx-2/3. Similarly, Pdx1 has also been shown to interact with $\mathrm{Cdx}-2 / 3$ in solution [49] thus potentially neutralizing $\mathrm{Cdx}-2 / 3$ function independent of DNA binding. In the same study, Pdx 1 was able to inhibit the $\mathrm{Cdx}-2 / 3$ mediated activation of the sucroseisomaltase gene in heterologous cells. (ii) Alternatively, free Pdx1 might efficiently compete for common coactivators and impair their interaction with DNAbound transcription factors thus blocking transcriptional activation. In this regard it is interesting that overexpression of Pdx1 in insulin-producing cells has recently been shown to repress insulin promoter activity [50]. Similarly, an excess of Pdx1 impaired its synergy with E47 on the insulin mini-enhancer in non-islet cells [16]. One common cofactor linking specific transcription factors to the basal transcription machinery, CBP/p300, is able to physically interact with Pax$6, \mathrm{Cdx}-2 / 3$, and $\mathrm{Pdx} 1[38,51]$. We therefore cotransfected BHK-21 cells with increasing amounts of $C B P$ or p300; however, this complementation with transcriptional coactivators failed to compensate for the transcriptional repression by Pdx1. Pax-6 contains an unusually long transactivation domain that has been reported to directly interact with the TATA binding protein within TFIID (TBP) [52]. Pdx 1 could thus impair Pax-6 interactions with TBP or alternatively, act by disturbing a multifactorial transcription complex by altering the ratio of its free components.

Pdx1 has been implicated in the tissue-specific activation of different genes through cell type and promoter context dependent protein to protein interactions. In acinar cells, $\mathrm{Pdx} 1$ associates with $\mathrm{Pbx} 1$ and Meis2 on the B element of the elastase I gene [40] and endocrine-specific complexes consist of $\mathrm{Pdx} 1 / \mathrm{Pbx}$, Pdx1/Pax-6 or E47/Beta2/Pdx1 on the somatostatin and insulin gene promoter, respectively $[13,14,15$, $16,17]$. These protein contacts allow for cooperative DNA binding and synergistic transcriptional activation. We show a new potential function of Pdx1 as mediator of cell-specific expression of the glucagon gene through its inhibition of Pax- 6 and Cdx-2/3 mediated transcription.

Acknowledgements. We thank V. Schwitzgebel, D. Drucker, H. Edlund, M.S. German, S. Saule, C.V.E. Wright, D. Melloul, N. Copeland, and M.D. Walker for generously providing molecular probes. This work was supported by the Swiss National Fund (J. Philippe, P.A. Halban), the Institute for Human Genetics and Biochemistry (J. Philippe), the Berger Foundation (J. Philippe), Juvenile Diabetes Research Foundation International (P.A. Halban) and the Carlos and Elsie de Reuters Foundation (J. Philippe).

\section{References}

1. Kim SK, Hebrok M, Melton DA (1997) Notochord to endoderm signaling is required for pancreas development. Development 124:4243-4252

2. Hebrok M, Kim SK, Melton DA (1998) Notochord repression of endodermal Sonic hedgehog permits pancreas development. Genes Dev 12:1705-1713 
3. Li H, Arber S, Jessell TM, Edlund (1999) Selective agenesis of the dorsal pancreas in mice lacking homeobox gene Hlxb9. Nat Genet 23:67-70

4. Harrison KA, Thaler J, Pfaff SL, Gu H, Kehrl, JH (1999) Pancreas dorsal lobe agenesis and abnormal islets of Langerhans in Hlxb9-deficient mice. Nat Genet 23:7175

5. Jonsson J, Carlsson L, Edlund T, Edlund H (1994) Insulinpromoter-factor 1 is required for pancreas development in mice. Nature 371:606-609

6. Ahlgren U, Jonsson J, Edlund H (1996) The morphogenesis of the pancreatic mesenchyme is uncoupled from that of the pancreatic epithelium in IPF1/PDX1-deficient mice. Development 122:1409-1416

7. Thor S, Ericson J, Brannstrom T, Edlund T (1991) The homeodomain LIM protein Isl-1 is expressed in subsets of neurons and endocrine cells in the adult rat. Neuron 7:881-889

8. Dong J, Asa SL, Drucker DJ (1991) Islet cell and extrapancreatic expression of the LIM domain homeobox gene isl-1. Mol Endocrinol 5:1633-1641

9. Ahlgren U, Pfaff SL, Jessell TM, Edlund T, Edlund H (1997) Independent requirement for ISL1 in formation of pancreatic mesenchyme and islet cells. Nature 385:257260

10. St-Onge L, Sosa-Pineda B, Chowdhury K, Mansouri A, Gruss P (1997) Pax6 is required for differentiation of glucagon-producing alpha-cells in mouse pancreas. Nature 387:406-409

11. Sander M, Neubüser A, Kalamaras J, Ee HC, Martin GR, German MS (1997) Genetic analysis reveals that PAX6 is required for normal transcription of pancreatic hormone genes and islet development. Genes Dev 11:1662-1673

12. Sosa-Pineda B, Chowdhury K, Torres M, Oliver G, Gruss P (1997) The Pax4 gene is essential for differentiation of insulin-producing beta cells in the mammalian pancreas. Nature 386:399-402

13. Peers B, Leonard J, Sharma S, Teitelman G, Montminy MR (1994) Insulin expression in pancreatic islet cells relies on cooperative interactions between the helix loop helix factor E47 and the homeobox factor STF-1. Mol Endocrinol 8:1798-1806

14. Peers B, Sharma S, Johnson T, Kamps M, Montminy M (1995) The pancreatic islet factor STF-1 binds cooperatively with $\mathrm{Pbx}$ to a regulatory element in the somatostatin promoter: importance of the FPWMK motif and of the homeodomain. Mol Cell Biol 15:7091-7097

15. Andersen FG, Jensen J, Heller RS et al. (1999) Pax6 and Pdx1 form a functional complex on the rat somatostatin gene upstream enhancer. FEBS Lett 445:315-320

16. Ohneda K, Mirmira RG, Wang J, Johnson JD, German MS (2000) The homeodomain of PDX-1 mediates multiple protein-protein interactions in the formation of a transcriptional activation complex on the insulin promoter. Mol Cell Biol 20:900-911

17. Glick E, Leshkowitz D, Walker MD (2000) Transcription factor BETA2 acts cooperatively with E2A and PDX1 to activate the insulin gene promoter. J Biol Chem 275:21992204

18. Schwartz PT, Perez-Villamil B, Rivera A, Moratalla R, Vallejo M (2000) Pancreatic homeodomain transcription factor IDX1/IPF1 expressed in developing brain regulates somatostatin gene transcription in embryonic neural cells. J Biol Chem 275:19106-19114

19. Ahlgren U, Jonsson J, Jonsson L, Simu K, Edlund H (1998) beta-cell-specific inactivation of the mouse Ipf1/
Pdx1 gene results in loss of the beta-cell phenotype and maturity onset diabetes. Genes Dev 12:1763-1768

20. Lottmann H, Vanselow J, Hessabi B, Walther R (2001) The Tet-On system in transgenic mice: inhibition of the mouse pdx-1 gene activity by antisense RNA expression in pancreatic beta-cells. J Mol Med 79:321-328

21. Wang H, Maechler P, Ritz-Laser B et al. (2001) Pdx1 level defines pancreatic gene expression pattern and cell lineage differentiation. J Biol Chem 276:25279-25286

22. Takaki R, Ono J, Nakmura M et al. (1986) Isolation of glucagon-secreting cell lines by cloning insulinoma cells. In Vitro Cell Dev Biol 22:120-126

23. Powers AC, Efrat S, Mojsov S, Spector D, Habener JF, Hanahan D (1990) Proglucagon processing similar to normal islets in pancreatic alpha-like cell line derived from transgenic mouse tumor. Diabetes 39:406-414

24. Santerre RF, Cook RA, Crisel RM et al. (1981) Insulin synthesis in a clonal cell line of simian virus 40-transformed hamster pancreatic beta cells. Proc Natl Acad Sci USA 78:4339-4343

25. Graham FL, Eb AJ van der (1973) Transformation of rat cells by DNA of human adenovirus. Virology 54:536-539

26. Henthorn P, Zervos P, Raducha M, Harris H, Kadesh T (1988) Expression of a human placental alkaline phosphatase gene in transfected cells: use as a reporter for studies of gene expression. Proc Natl Acad Sci USA 85:63426346

27. Drucker DJ, Philippe J, Jepeal L, Habener JF (1987) Glucagon gene 5'-flanking sequences promote islet cell-specific gene transcription. J Biol Chem 262:15659-15665

28. Morel C, Cordier-Bussat M, Philippe J (1995) The upstream promoter element of the glucagon gene, G1, confers pancreatic alpha cell-specific expression. J Biol Chem 270:3046-3055

29. Philippe J, Missotten M (1990) Functional characterization of a cAMP-responsive element of the rat insulin I gene. J Biol Chem 265:1465-1469

30. Zufferey R, Nagy D, Mandel R J, Naldini L, Trono D (1997) Multiply attenuated lentiviral vector achieves efficient gene delivery in vivo. Nat Biotechnol 15:871-875

31. Naldini L, Blomer U, Gallay P et al. (1996) In vivo gene delivery and stable transduction of nondividing cells by a lentiviral vector. Science 272:263-267

32. Salmon P, Kindler V, Ducrey O, Chapuis B, Zubler RH, Trono D (2000) High-level transgene expression in human hematopoietic progenitors and differentiated blood lineages after transduction with improved lentiviral vectors. Blood 96:3392-3398

33. Schreiber E, Mathias P, Muller MM, Schaffner W (1988) Identification of a novel lymphoid specific octamer binding protein (OTF-2B) by proteolytic clipping bandshift assay (PCBA). EMBO J 7:4221-4229

34. Philippe J (1991) Insulin regulation of the glucagon gene is mediated by an insulin-responsive DNA element. Proc Natl Acad Sci USA 88:7224-7227

35. Laser B, Meda P, Constant I, Philippe J (1996) The caudalrelated homeodomain protein $\mathrm{Cdx}-2 / 3$ regulates glucagon gene expression in islet cells. J Biol Chem 271:28984-28994

36. Ritz-Laser B, Estreicher A, Klages N, Saule S, Philippe J (1999) Pax-6 and Cdx-2/3 interact to activate glucagon gene expression on the G1 control element. J Biol Chem 274:4124-4132

37. Emens LA, Landers DW, Moss LG (1992) Hepatocyte nuclear factor 1 alpha is expressed in a hamster insulinoma line and transactivates the rat insulin I gene. Proc Natl Acad Sci USA 89:7300-7304 
38. Hussain MA, Habener JF (1999) Glucagon gene transcription activation mediated by synergistic interactions of pax6 and cdx-2 with the p300 co-activator. J Biol Chem 274:28950-28957

39. Wang M, Drucker DJ (1995) The LIM domain homeobox gene isl- 1 is a positive regulator of islet cell-specific proglucagon gene transcription. J Biol Chem 270:12646-12652

40. Swift GH, Liu Y, Rose SD et al. (1998) An endocrine-exocrine switch in the activity of the pancreatic homeodomain protein PDX1 through formation of a trimeric complex with PBX1b and MRG1 (MEIS2). Mol Cell Biol 18:5109_ 5120

41. Jin T, Drucker D (1996) Activation of proglucagon gene transcription through a novel promoter element by the caudal-related homeodomain protein cdx-2/3. J Mol Cell Biol 16:19-28

42. Miller CP, McGhee Jr RE, Habener JF (1994) IDX-1: a new homeodomain transcription factor expressed in rat pancreatic islets and duodenum that transactivates the somatostatin gene. EMBO J 13:1145-1156

43. Gauthier BR, Schwitzgebel VM, Zaiko M, Mamin A, Ritz-Laser B, Philippe J (2002) Hepatic nuclear factor-3 (HNF-3 or Foxa2) regulates glucagon gene transcription by binding to the G1 and G2 Promoter Elements. Mol Endocrinol 16:170-183

44. Herzig S, Fuzesi L, Knepel W (2000) Heterodimeric PbxPrep1 homeodomain protein binding to the glucagon gene restricting transcription in a cell type-dependent manner. J Biol Chem 275:27989-27999
45. Lu M, Miller C, Habener J (1996) Functional regions of the homeodomain protein IDX-1 required for transactivation of the rat somatostatin gene. Endocrinology 137:29592967

46. Serup P, Jensen J, Andersen FG et al. (1996) Induction of insulin and islet amyloid polypeptide production in pancreatic islet glucagonoma cells by insulin promoter factor 1 . Proc Natl Acad Sci USA 93:9015-9020

47. Watada H, Kajimoto Y, Miyagawa J et al. (1996) PDX-1 induces insulin and glucokinase gene expressions in alphaTC1 clone 6 cells in the presence of betacellulin. Diabetes 45:1826-1831

48. Elrick LJ, Docherty K (2001) Phosphorylation-dependent nucleocytoplasmic shuttling of pancreatic duodenal homeobox-1. Diabetes 50:2244-2252

49. Heller RS, Stoffers DA, Hussain MA, Miller CP, Habener JF (1998) Misexpression of the pancreatic homeodomain protein IDX-1 by the Hoxa-4 promoter associated with agenesis of the cecum. Gastroenterology 115:381-387

50. Seijffers R, Ben-David O, Cohen Y et al. (1999) Increase in PDX-1 levels suppresses insulin gene expression in RIN 1046-38 cells. Endocrinology 140:3311-3317

51. Qiu Y, Guo M, Huang S, Stein R (2002) Insulin gene transcription is mediated by interactions between the p300 coactivator and PDX-1, BETA2, and E47. Mol Cell Biol 22:412-420

52. Cvekl A, Kashanchi F, Brady JN, Piatigorsky J (1999) Pax-6 interactions with TATA-box-binding protein and retinoblastoma protein. Invest Ophthalmol Vis Sci 40:1343-1350 